ARTICLE OPEN

\title{
Formation of environmentally stable hole-doped graphene films with instantaneous and high-density carrier doping via a boron-based oxidant
}

\author{
Kaito Kanahashi ${ }^{1}$, Naoki Tanaka ${ }^{2}$, Yoshiaki Shoji $\mathbb{D}^{2}$, Mina Maruyama ${ }^{3}$, II Jeon ${ }^{4}$, Kenji Kawahara ${ }^{5}$, Masatou Ishihara ${ }^{6}$, Masataka \\ Hasegawa $^{6}$, Hiromichi Ohta ${ }^{7}{ }^{7}$, Hiroki Ago $\mathbb{D}^{5}$, Yutaka Matsuo $\mathbb{D i D}^{4,8}$, Susumu Okada ${ }^{3}$, Takanori Fukushima (D) $^{2}$ and Taishi Takenobu ${ }^{1,9}$
}

Large-area graphene films have substantial potential for use as next-generation electrodes because of their good chemical stability, high flexibility, excellent carrier mobility, and lightweight structure. However, various issues remain unsolved. In particular, highdensity carrier doping within a short time by a simple method, and air stability of doped graphene films, are highly desirable. Here, we demonstrate a solution-based high-density $\left(>10^{14} \mathrm{~cm}^{-2}\right)$ hole doping approach that promises to push the performance limit of graphene films. The reaction of graphene films with a tetrakis(pentafluorophenyl)borate salt, containing a two-coordinate boron cation, achieves doping within an extremely short time (4s), and the doped graphene films are air stable for at least 31 days. X-ray photoelectron spectroscopy reveals that the graphene films are covered by the chemically stable anions, resulting in an improved stability in air. Moreover, the doping reduces the transmittance by only $0.44 \pm 0.23 \%$. The simplicity of the doping process offers a viable route to the large-scale production of functional graphene electrodes.

npj 2D Materials and Applications (2019)3:7; https://doi.org/10.1038/s41699-019-0090-x

\section{INTRODUCTION}

Large-area graphene films obtained by a chemical vapor deposition (CVD) growth method are transferable onto any substrate, including flexible and stretchable materials. ${ }^{1-4}$ These films have therefore opened a path for the development of large-area electrical and optical applications, including electrodes for Li-ion batteries, ${ }^{5,6}$ supercapacitors, ${ }^{7,8}$ and flexible transparent conducting films, ${ }^{9-11}$ because of their good chemical stability, high flexibility, excellent carrier mobility, and lightweight structures. ${ }^{12-17}$ However, various problems remain to be solved to enable the practical application of large-area graphene electrodes. The most important issues to consider when using graphene as an electrode are (i) enhancing the electrical conductivity by carrier doping, (ii) improving the long-term stability of the doped graphene films in ambient air, (iii) ensuring compatibility with a facile solution-based doping process at room temperature and with a short process time, and (iv) maintaining a clean film surface after doping to avoid surface contaminants that may reduce film transparency and increase contact resistance. To date, numerous accounts of the chemical doping of graphene have been reported. ${ }^{3,18-29}$ However, none of them fulfills all four of the aforementioned requirements.

Given these obstacles, we focused on the salt of a twocoordinate boron cation, $\mathrm{Mes}_{2} \mathrm{~B}^{+}$(Mes = 2,4,6-trimethylphenyl group), with a chemically stable tetrakis(pentafluorophenyl)borate anion $\left[\left(C_{6} F_{5}\right)_{4} B\right]^{-}$(Fig. 1a). ${ }^{30}$ We recently investigated the solutionbased hole doping of single-walled carbon nanotubes (CNTs) ${ }^{31}$ and transition metal dichalcogenide monolayers ( $\mathrm{WSe}_{2}$ monolayers $)^{32}$ using $\mathrm{Mes}_{2} \mathrm{~B}^{+}\left[\left(\mathrm{C}_{6} \mathrm{~F}_{5}\right)_{4} \mathrm{~B}\right]^{-}$and found that efficient hole doping of CNT films and WSe $\mathrm{W}_{2}$ monolayers was achieved with this reagent because of the electron-deficient nature of $\mathrm{Mes}_{2} \mathrm{~B}^{+} .{ }^{30,33}$ In the hole-doped CNTs and WSe 2 monolayers, $\left[\left(\mathrm{C}_{6} \mathrm{~F}_{5}\right)_{4} \mathrm{~B}\right]^{-}$anions compensated for the positive charge generated by the hole doping. Notably, $\left[\left(\mathrm{C}_{6} \mathrm{~F}_{5}\right)_{4} \mathrm{~B}\right]^{-}$anions covered the surface of the doped materials, enhancing their stability in ambient air because of the anions' chemically inert, thermally stable, and hydrophobic nature. ${ }^{34}$ As a result, a highly air-stable and moisture-stable doped state of the CNTs was achieved by a solution-based process. Therefore, $\mathrm{Mes}_{2} \mathrm{~B}^{+}\left[\left(\mathrm{C}_{6} \mathrm{~F}_{5}\right)_{4} \mathrm{~B}\right]^{-}$is expected to function as a molecular dopant for graphene, potentially satisfying the four aforementioned requirements.

In this study, the chemical hole doping of large-area graphene films produced using a CVD method is experimentally and theoretically investigated. First, we reacted graphene thin films with $\mathrm{Mes}_{2} \mathrm{~B}^{+}\left[\left(\mathrm{C}_{6} \mathrm{~F}_{5}\right)_{4} \mathrm{~B}\right]^{-}$and characterized the resultant-doped graphene films by four-probe sheet resistance, transmittance, $\mathrm{X}$-ray photoelectron spectroscopy, thermopower, and Hall effect measurements after rinsing them with an organic solvent. We observe a high carrier density of approximately $2.57 \pm 0.98 \times 10^{14} \mathrm{~cm}^{-2}$ as a result of the 4-s solution-based doping method, and the transmittance reduction at $550 \mathrm{~nm}$ caused by doping is only $0.44 \pm 0.23 \%$. The doped state is maintained for at least 31 days in air without a substantial change in resistance. In addition, we

\footnotetext{
${ }^{1}$ Department of Advanced Science and Engineering, Waseda University, Tokyo 169-8555, Japan; ${ }^{2}$ Laboratory for Chemistry and Life Science, Institute of Innovative Research, Tokyo Institute of Technology, Yokohama 226-8503, Japan; ${ }^{3}$ Graduate School of Pure and Applied Science, University of Tsukuba, Ibaraki 305-8571, Japan; ${ }^{4}$ Department of Mechanical Engineering, School of Engineering, The University of Tokyo, Tokyo 113-8565, Japan; ${ }^{5}$ Global Innovation Center (GIC), Kyushu University, Fukuoka 816-8580, Japan; ${ }^{6}$ Carbon-Based Thin Film Materials Group, National Institute of Advanced Industrial Science and Technology (AIST), Ibaraki 305-8565, Japan; ${ }^{7}$ Research Institute for Electronic

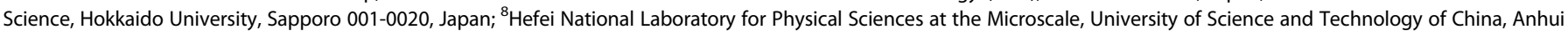
230026, China and ${ }^{9}$ Department of Applied Physics, Nagoya University, Nagoya 464-8603, Japan

Correspondence: Taishi Takenobu (takenobu@nagoya-u.jp)
}

Received: 8 September 2018 Accepted: 10 January 2019

Published online: 01 February 2019 
(a)
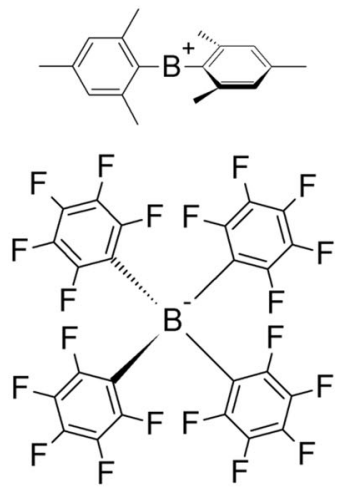

(b)

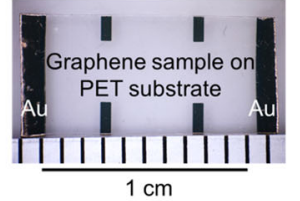

Graphene sample on glass substrate

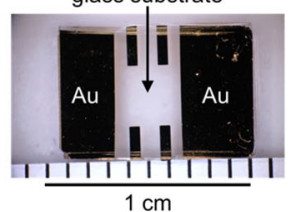

(c)

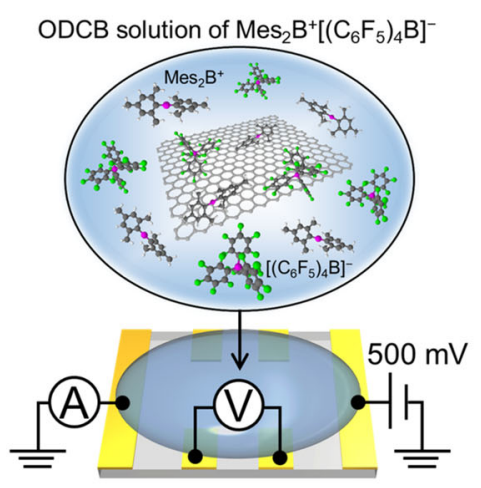

(d)

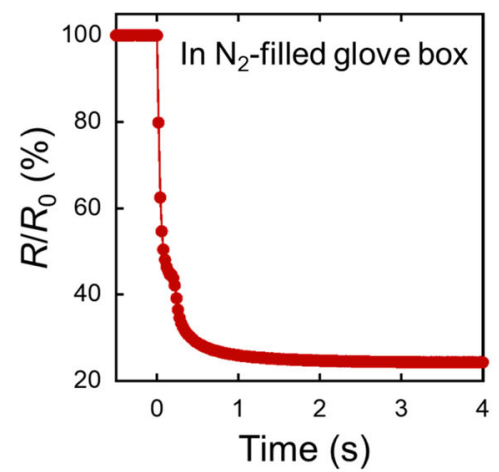

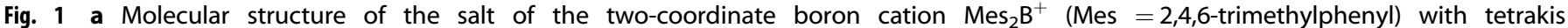

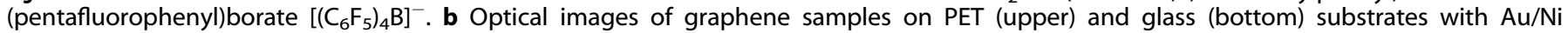

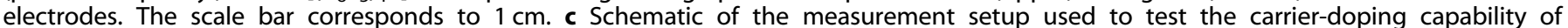

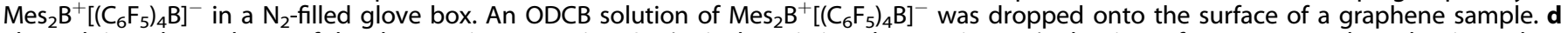

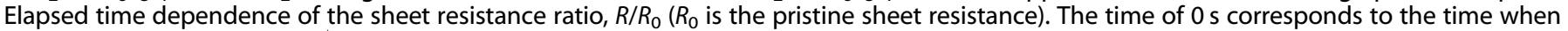
the ODCB solution of $\mathrm{Mes}_{2} \mathrm{~B}^{+}\left[\left(\mathrm{C}_{6} \mathrm{~F}_{5}\right)_{4} \mathrm{~B}\right]^{-}$was dropped

demonstrated the doped graphene films' applicability in organic photovoltaic cells, in which they were used as the transparent electrodes. Finally, we performed energy-band calculations of holedoped graphene with $\left[\left(\mathrm{C}_{6} \mathrm{~F}_{5}\right)_{4} \mathrm{~B}\right]^{-}$anions by the density functional theory (DFT) method and confirmed the high stability of the holedoped graphene- $\left[\left(\mathrm{C}_{6} \mathrm{~F}_{5}\right)_{4} \mathrm{~B}\right]^{-}$complex.

\section{RESULTS}

Instantaneous carrier doping inside a nitrogen-filled glove box We used large-area graphene films produced by the surface wave plasma CVD method. ${ }^{4,35}$ The graphene films were synthesized on $\mathrm{Cu}$ foils $(23 \mathrm{~cm} \times 20 \mathrm{~cm} \times 33 \mu \mathrm{m}$ thick) and transferred onto two types of substrates: polyethylene terephthalate (PET) (188 $\mu \mathrm{m}$ thick) and glass ( $1 \mathrm{~mm}$ thick). Notably, the resultant graphene films are not singlelayer graphene; the average layer number estimated from Raman spectroscopy is two (see supplementary S1). As shown in Fig. 1b, we cut the large-area graphene film into smaller pieces, and $\mathrm{Au} / \mathrm{Ni}$ $(80 \mathrm{~nm} / 3 \mathrm{~nm})$ electrodes were thermally deposited onto the graphene samples. For the solution-based doping process with $\mathrm{Mes}_{2} \mathrm{~B}^{+}\left[\left(\mathrm{C}_{6} \mathrm{~F}_{5}\right)_{4} \mathrm{~B}\right]^{-}$, we prepared a saturated o-dichlorobenzene (ODCB) solution of $\mathrm{Mes}_{2} \mathrm{~B}^{+}\left[\left(\mathrm{C}_{6} \mathrm{~F}_{5}\right)_{4} \mathrm{~B}\right]^{-}(30 \mathrm{mg} / \mathrm{mL})$ in a $\mathrm{N}_{2}$-filled glove box at room temperature because $\mathrm{Mes}_{2} \mathrm{~B}^{+}$is immediately hydrolyzed upon exposure to ambient air. ${ }^{30}$ Then, as shown in Fig. 1C, we dropped the ODCB solution of $\mathrm{Mes}_{2} \mathrm{~B}^{+}\left[\left(\mathrm{C}_{6} \mathrm{~F}_{5}\right)_{4} \mathrm{~B}\right]^{-}$onto the graphene samples on a PET substrate and measured their four-probe sheet resistance $(R)$ to test the carrier-doping capability of $\mathrm{Mes}_{2} \mathrm{~B}^{+}\left[\left(\mathrm{C}_{6} \mathrm{~F}_{5}\right)_{4} \mathrm{~B}\right]^{-}$. For measurements of $R$, we applied a constant voltage of $500 \mathrm{mV}$ and estimated $R$ from the collected currents using a semiconductor parameter analyzer (Agilent B2902A). Figure 1d shows the elapsed time dependence of the sheet resistance ratio, $R$ / $R_{0}$ ( $R_{0}$ is the resistance of a pristine sample), where the time of $0 \mathrm{~s}$ corresponds to the time when the ODCB solution of $\mathrm{Mes}_{2} \mathrm{~B}^{+}\left[\left(\mathrm{C}_{6} \mathrm{~F}_{5}\right)_{4} \mathrm{~B}\right]^{-}$was dropped onto the graphene samples. The observed rapid decrease in $R / R_{0}$ is a clear signature of carrier doping of $\mathrm{Mes}_{2} \mathrm{~B}^{+}\left[\left(\mathrm{C}_{6} \mathrm{~F}_{5}\right)_{4} \mathrm{~B}\right]^{-}$into the graphene films; the achieved $R / R_{0}$ of $24.2 \%$ is similar to the previously reported lowest best value $(23.9 \%)^{25}$ among solution-based doping methods, indicating the efficient carrier doping by $\operatorname{Mes}_{2} \mathrm{~B}^{+}\left[\left(\mathrm{C}_{6} \mathrm{~F}_{5}\right)_{4} \mathrm{~B}\right]^{-}$. Moreover, the decrease in $R / R_{0}$ was saturated within a short time $(\sim 4 \mathrm{~s})$, which is substantially faster than previously reported hole-doping times $(60 \mathrm{~min}){ }^{24}$ It should be noted that we performed the same experiments using a lower density ODCB solution of $\operatorname{Mes}_{2} B^{+}\left[\left(C_{6} F_{5}\right)_{4} B\right]^{-}(0.3 \mathrm{mg} / \mathrm{mL})$, and the necessary doping time is very similar (see supplementary S2), also revealing efficient carrier doping by $\operatorname{Mes}_{2} B^{+}\left[\left(C_{6} F_{5}\right)_{4} B\right]^{-}$. However, the achieved $R / R_{0}$ by the lower density ODCB solution (35.0\%) is slightly higher than that of saturated ODCB solution (see supplementary S2). These results suggest the possible resistance controllability of this doping method.

Stability of doped graphene films in ambient air

Next, to investigate the doping capability of $\operatorname{Mes}_{2} \mathrm{~B}^{+}\left[\left(\mathrm{C}_{6} \mathrm{~F}_{5}\right)_{4} \mathrm{~B}\right]^{-}$in greater detail, we modified the doping method by introducing an additional rinsing process to clean the surface of the doped graphene films in a $\mathrm{N}_{2}$-filled glove box and conducted measurements in ambient air to examine the air stability of the doped graphene films. Figure $2 a$ shows a schematic of the sample preparation process in a $\mathrm{N}_{2}$-filled glove box. First, graphene samples were immersed in a saturated ODCB solution of $\mathrm{Mes}_{2} \mathrm{~B}^{+}\left[\left(\mathrm{C}_{6} \mathrm{~F}_{5}\right)_{4} \mathrm{~B}\right]^{-}$for $1 \mathrm{~min}$, which is longer than the time required for carrier doping $(4 \mathrm{~s})$. The graphene samples were then removed from the ODCB solution and rinsed with dry ODCB to clean the surface of the samples. Finally, the doped graphene samples were annealed at $60^{\circ} \mathrm{C}$ for $10 \mathrm{~min}$ on a hotplate to remove any residual ODCB. These doping, rinsing and annealing procedures were performed in a $\mathrm{N}_{2}$-filled glove box at room temperature since $\mathrm{Mes}_{2} \mathrm{~B}^{+}$is unstable in air. ${ }^{30}$ We characterized the effect of the rinsing process using atomic force microscopy (AFM, C3000, Nanosurf) in ambient air. As shown in Fig. 2b, the topography of graphene films is very similar before and after the doping process, suggesting the surface of the samples is cleaned well and the dopant layer is molecularly flat. The sheet resistance and transmittance of the graphene samples were then measured in ambient air without any atmospheric regulation.

Figure 2c shows the current $(I)$-voltage $(V)$ characteristics of pristine (gray) and doped (red) graphene samples on a glass substrate (Sample \#1). The sheet resistance $(R)$ was measured using a source meter (Agilent, B2902A) and a nanovoltmeter (Keysight, 34420A) in ambient air. The $R$ value of the graphene sample decreases substantially from $837 \Omega /$ sq to $207 \Omega / s q$, and the resulting $R / R_{0}$ value of $24.7 \%$ measured in ambient air is very similar to that measured inside the glove box (24.2\%). Therefore, these results strongly suggest that the graphene films doped with Mes $_{2} \mathrm{~B}^{+}\left[\left(\mathrm{C}_{6} \mathrm{~F}_{5}\right)_{4} \mathrm{~B}\right]^{-}$are highly air stable. Seven independently prepared graphene samples on glass substrates were measured before and after doping, and the doping effect is highly 
(a)

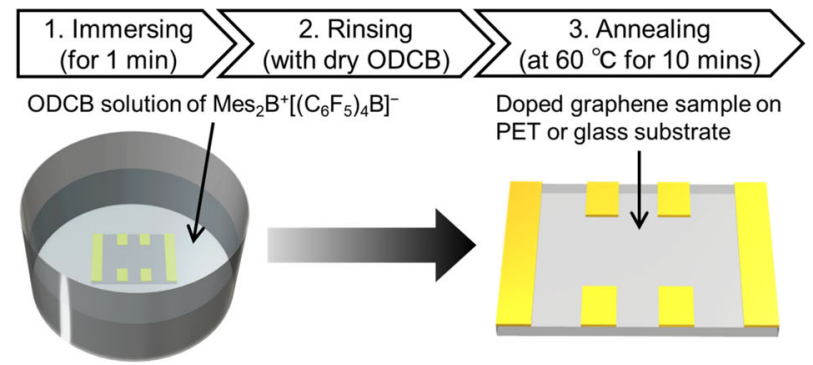

(b)

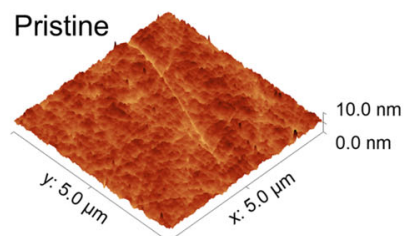

RMS: $0.28 \pm 0.03 \mathrm{~nm}$ (c)

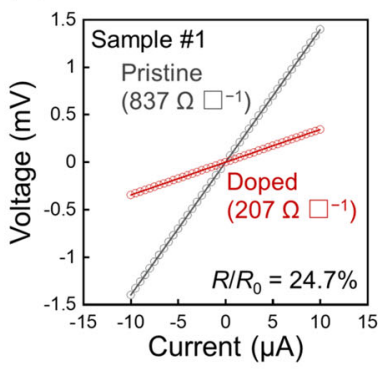

(e)

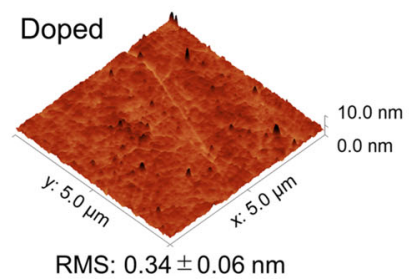

(d)
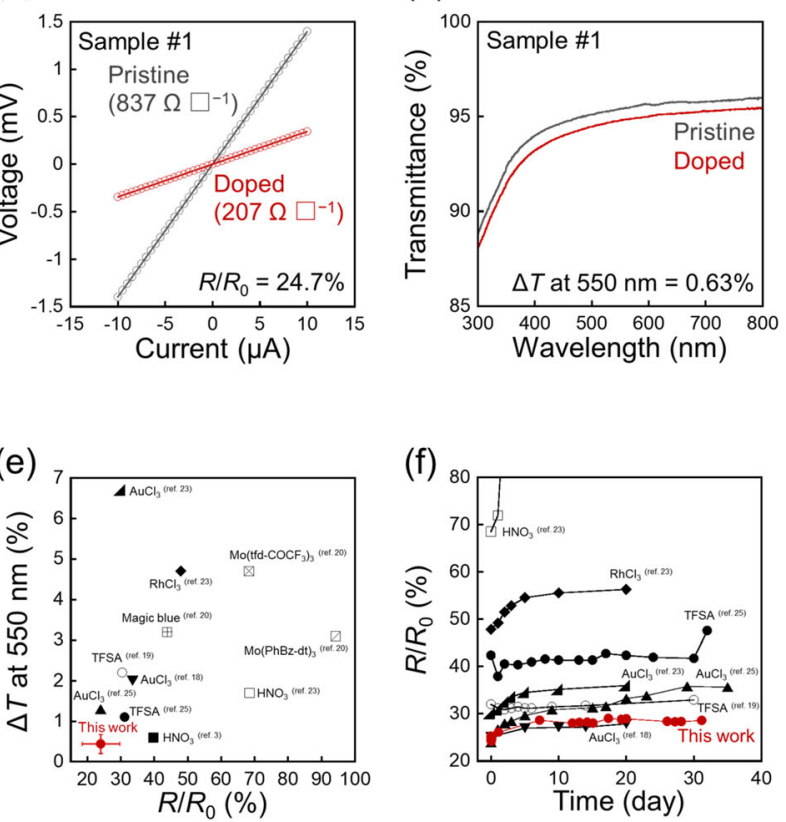

(f)

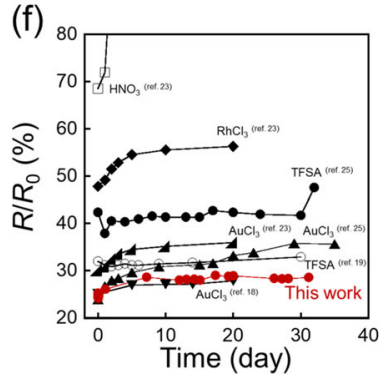

Fig. 2 a Schematic of the doping procedure, which involves rinsing of the doped graphene film by dry ODCB. b AFM images of pristine and doped graphene films. The root mean square (RMS) values of each film are also displayed. c Current-voltage characteristics of pristine (gray) and doped (red) graphene samples on a glass substrate (Sample \#1). d Optical transmittance spectra of pristine (gray) and doped (red) graphene samples on a glass substrate (Sample \#1). e A summary of the relation between the transmittance decrease $(\Delta T)$ at $550 \mathrm{~nm}$ and the sheet resistance ratio $R / R_{0}$. The red circle corresponds to the results of this work. The results from previous reports are also plotted. $f$ Timedependent changes in ambient air of the sheet resistance ratio $R / R_{0}$. The results from previous reports using other dopants $\left(\mathrm{AuCl}_{3}\right.$, $\mathrm{HNO}_{3}$, $\mathrm{RhCl}_{3}$, and TFSA) are also plotted

reproducible (see supplementary S3 (Table S1)). It should be noted that, in a $\mathrm{N}_{2}$-filled glove box, we also measured the resistances of the pristine sample, doped sample before rinsing, and doped sample after rinsing. Importantly, the film resistances of the doped sample before rinsing and doped sample after rinsing are very similar, suggesting the strong interaction between the dopant and graphene film (see supplementary S3).

As the next step, we investigated the effect of carrier doping on film transparency because, in most transparent electrodes, the film conductivity and transparency typically follow opposite trends. $^{3,18-20,22-26,28}$ Figure $2 d$ shows transmission spectra of the pristine (gray) and doped (red) graphene samples on a glass substrate (Sample \#1); these samples were used for $I-V$ measurements (Fig. 2c). In Fig. 2d, the doped graphene sample barely shows a decrease in transmittance $(\Delta T)$. Reproducible results for $\Delta T$ at $550 \mathrm{~nm}$ were obtained for five independently prepared graphene samples on a glass substrate, and the average reduction in transmittance at $550 \mathrm{~nm}$ is $0.44 \pm 0.23 \%$ (see supplementary S3 (Table S1)). To the best of our knowledge, the observed average $\Delta T$ of $0.44 \pm 0.23 \%$ at $550 \mathrm{~nm}$ is the lowest minimum value among those reported for graphene films obtained by a solution-based doping process. ${ }^{3,18-20,22-25}$

These results suggest that, during the doping process, the rinsing with ODCB removes contaminants that adversely affect the transmittance of the graphene films. To confirm the effect of the rinsing process on sample transmittance, we also compared unrinsed doped graphene samples with rinsed doped samples on a PET substrate (see supplementary S4 (Table S2)). For the unrinsed doped samples, the transmittances of five independently prepared samples were measured; the average $\Delta T$ at $550 \mathrm{~nm}$ is $6.67 \pm 6.55 \%$. However, in sharp contrast to the case of the unrinsed doped samples, the average $\Delta T$ of the rinsed doped graphene samples is only $1.04 \pm 0.86 \%$ (see supplementary S4 (Table S2)). Therefore, we conclude that the rinsing process effectively removes surface contaminants and keeps the sample surface clean, resulting in high transmittance with a low sheet resistance. Figure 2e summarizes the $\Delta T$ values at $550 \mathrm{~nm}$ and the sheet resistance ratio $R / R_{0}$ for the doped graphene samples prepared by solution-based doping methods, as obtained from both the present study and the literature (see supplementary S5 (Table S3)). ${ }^{3,18,19,23-25}$ The graphene samples doped with $\mathrm{Mes}_{2} \mathrm{~B}^{+}\left[\left(\mathrm{C}_{6} \mathrm{~F}_{5}\right)_{4} \mathrm{~B}\right]^{-}$are found to possess the highest levels of both optical transparency and electrical conductivity.

As previously discussed, these results also suggest that the graphene films doped with $\operatorname{Mes}_{2} \mathrm{~B}^{+}\left[\left(\mathrm{C}_{6} \mathrm{~F}_{5}\right)_{4} \mathrm{~B}\right]^{-}$are highly air stable; therefore, we investigated the long-term air stability of the doped graphene samples. Figure $2 f$ shows the time-dependent changes in the $R / R_{0}$ value. If we consider that the carrier mobility of doped samples is sufficiently similar to that of pristine samples, then, according to the Drude model, the plot in Fig. $2 f$ correlates directly with the time-dependent change in carrier density. Figure 2f shows a comparison of the results for the currently doped graphene sample with those for graphene using previously reported solution-based hole-doping reagents under ambient conditions (see supplementary S6 (Table S4)). ${ }^{18,19,23,25}$ After 31 days, we observe that the $R / R_{0}$ value of the graphene sample doped with $\mathrm{Mes}_{2} \mathrm{~B}^{+}\left[\left(\mathrm{C}_{6} \mathrm{~F}_{5}\right)_{4} \mathrm{~B}\right]^{-}$increased from $24.7 \%$ to only $28.6 \%$. As evidenced by the results shown in Fig. 2, $\mathrm{Mes}_{2} \mathrm{~B}^{+}\left[\left(\mathrm{C}_{6} \mathrm{~F}_{5}\right)_{4} \mathrm{~B}\right]^{-}$is a new class of hole-doping reagent that can fulfill four important requirements of graphene doping: a high carrier density, the air stability of doped graphene, a facile solution-based doping process, and negligible transmittance reduction after doping. Moreover, we successfully fabricated organic photovoltaic cells (OPVs) ${ }^{28,29,36}$ using the Mes $_{2} \mathrm{~B}^{+}\left[\left(\mathrm{C}_{6} \mathrm{~F}_{5}\right)_{4} \mathrm{~B}\right]^{-}$-doped graphene as electrodes; the resultant devices exhibit an enhanced device performance in terms of the fill factor (FF) and power conversion efficiency (PCE). As shown in supplementary S7 (Fig. S4 and Table S5), the FF and PCE values for an OPV with a pristine double-layer graphene (DLG)-based anode are $0.48 \%$ and $4.34 \%$, respectively, whereas a device with a doped 
(a)

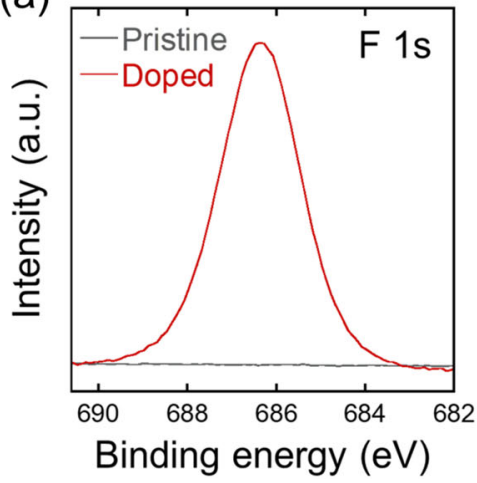

(b)

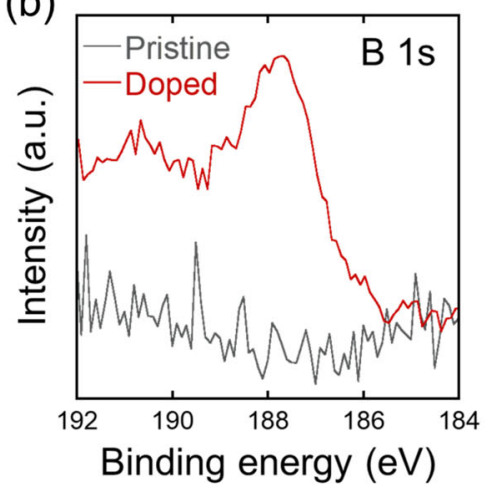

(c)

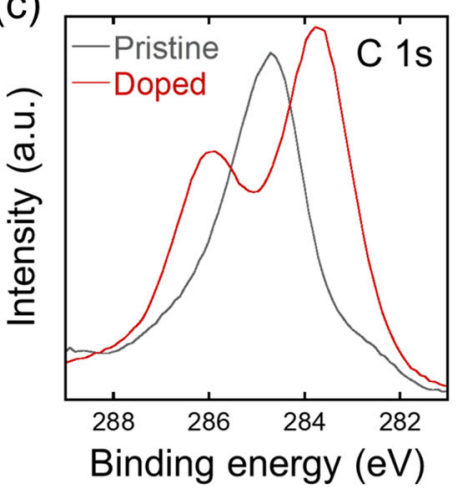

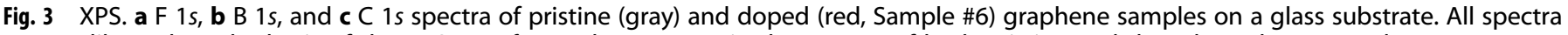
were calibrated on the basis of the XPS Au $4 f_{7 / 2}$ peak at $84.0 \mathrm{eV}$ in the spectra of both pristine and doped graphene samples

DLG shows improved FF (0.52) and PCE (4.73\%) values. The positive effect of doping on the device performance is also observed for single-layer graphene (SLG)-based OPVs (Fig. S4 and Table S5).

\section{X-ray photoelectron spectroscopy}

We performed X-ray photoelectron spectroscopy (XPS) to confirm the surface coverage of the doped graphene samples. Because we used the samples with gold electrodes, the binding energy was calibrated on the basis of the XPS Au $4 f_{7 / 2}$ peak at $84.0 \mathrm{eV}$ (see supplementary S8). Figure $3 a$ and $b$ shows XPS F $1 \mathrm{~s}$ and $B$ 1s peaks, respectively, of pristine and doped graphene samples on a glass substrate. Although both the XPS F 1s and XPS B 1s peaks are clearly observed in the spectra of the doped graphene sample (Sample \#6), the intensities of these peaks are negligible in the spectra of the pristine sample. Therefore, the surface of the doped graphene film should be covered by $\left[\left(\mathrm{C}_{6} \mathrm{~F}_{5}\right)_{4} \mathrm{~B}\right]^{-}$molecules, and we can conclude that these spectra provide solid evidence of holecarrier doping in the graphene films. Moreover, the positive charge carriers generated upon hole doping must be compensated by an equivalent amount of highly hydrophobic $\left[\left(\mathrm{C}_{6} \mathrm{~F}_{5}\right)_{4} \mathrm{~B}\right]^{-}$ molecules, which will likely keep components of the ambient atmosphere (such as $\mathrm{O}_{2}$ or $\mathrm{H}_{2} \mathrm{O}$ ) away from the graphene film to provide an air stability for the doped state of the film. These results also agree with previous reports on CNTs and $\mathrm{WSe}_{2}$ monolayers. ${ }^{31,32}$

As shown in Fig. 3c, we collected the XPS C 1s spectra of both pristine and doped samples. We observe a single XPS C $1 s$ peak at $284.8 \mathrm{eV}$ in the spectrum of the pristine sample, which reasonably agrees with the previously reported results. ${ }^{37}$ However, two XPS C $1 s$ peaks are obtained at $285.9 \mathrm{eV}$ and $283.8 \mathrm{eV}$ in the spectrum of the doped sample. Because the hole-carrier doping leads to a lowering of the Fermi energy, the lower-energy peak at $283.8 \mathrm{eV}$ is reasonably assigned as the contribution from the hole-doped graphene film. ${ }^{20,24}$ Moreover, the higher-energy peak at $285.9 \mathrm{eV}$ is assigned as the XPS $C 1 s$ peak of $\left[\left(C_{6} F_{5}\right)_{4} B\right]$ molecules because, in general, the binding energy of the XPS C 1s peak becomes larger when carbon forms a chemical bond with a highly electronegative atom, ${ }^{38,39}$ such as in the case of the $\mathrm{C}-\mathrm{F}$ bonds of $\left[\left(\mathrm{C}_{6} \mathrm{~F}_{5}\right)_{4} \mathrm{~B}\right]$ molecules. From the relative intensity ratio of the XPS $C 1 s$ and $B 1 s$ peaks, we obtain a $C / B$ atomic ratio of $61.5: 1$; these results correspond to a dopant density of $2.0 \times 10^{14} \mathrm{~cm}^{-2}$ for DLG. ${ }^{40}$ It should be noted that we can also estimate the defect density from the ratio between the $D$ and $G$ peak intensities in Raman spectra. ${ }^{41}$ This analysis results in a defect density of $3.85 \pm$ $1.07 \times 10^{10} \mathrm{~cm}^{-2}$ using the Raman spectra presented in Fig. S1 and indicates that the doping is not controlled by the initial defect density. If we assume that one hole carrier is compensated by one $\left[\left(\mathrm{C}_{6} \mathrm{~F}_{5}\right)_{4} \mathrm{~B}\right]^{-}$molecule, the obtained surface density of the
$\left[\left(\mathrm{C}_{6} \mathrm{~F}_{5}\right)_{4} \mathrm{~B}\right]^{-}$molecules corresponds to the doped hole density. Notably, to the best of our knowledge, a doped hole density greater than $10^{14} \mathrm{~cm}^{-2}$ is the highest hole density reported for graphene films obtained by solution-based carrier doping. ${ }^{19,23-25}$

Thermopower and the Hall effect measurements

Although XPS measurements indicate a high hole-carrier density greater than $10^{14} \mathrm{~cm}^{-2}$, we also investigated the carrier type and carrier density of doped samples using different methods. As shown in Fig. 4a, we measured the thermopower by applying a temperature gradient between two electrodes on a graphene sample using two Peltier elements (Laird Technologies CP1.0-31-05L). These Peltier elements were wired in series with a DC power supply (KEYSIGHT E3642A), and their temperature difference was monitored using a temperature controller (Lakeshore model 335) and alumel-chromel thermocouples (SAKAGUCHI E.H VOC CORP. TCKT0051). The thermoelectromotive force was measured using a voltmeter (KEYSIGHT 34420A). As shown in Fig. $4 \mathrm{~b}$, a positive relation between the thermoelectromotive force and the temperature difference of both pristine and doped graphene films is observed, indicating a positive thermopower. Therefore, the carrier type of graphene films doped with $\operatorname{Mes}_{2} B^{+}\left[\left(C_{6} F_{5}\right)_{4} B\right]^{-}$is unambiguously determined to be holes. ${ }^{42}$

Along with the thermopower measurements, Hall effect measurements (Fig. 4c, Quantum Design, PPMS) confirm that the charge carriers generated by doping with $\mathrm{Mes}_{2} \mathrm{~B}^{+}\left[\left(\mathrm{C}_{6} \mathrm{~F}_{5}\right)_{4} \mathrm{~B}\right]^{-}$ are holes, which agrees with the results of the XPS measurements. These measurements also allow for a quantitative evaluation of the carrier density. Figure $4 d$ shows the magnetic-field dependences of the Hall resistance $\left(R_{\text {Hall }}\right)$ of both pristine and doped graphene samples. A positive relation between the magnetic field and the Hall resistance is observed. This positive relation is clear evidence of the hole doping of the graphene film. ${ }^{42}$ On the basis of this relation, the hole-carrier density is determined to be $2.3 \times$ $10^{14} \mathrm{~cm}^{-2}$, which is virtually identical to that estimated by the XPS measurements $\left(2.0 \times 10^{14} \mathrm{~cm}^{-2}\right)$. Reproducible results for the doped hole density were obtained with other samples, and the average hole density is $2.57 \pm 0.98 \times 10^{14} \mathrm{~cm}^{-2}$, (see supplementary S3 (Table S1)). Again, we note that a doped hole density of more than $10^{14} \mathrm{~cm}^{-2}$ is the largest hole density reported to date for a doped graphene film obtained by a solution-based doping process. $^{19,23,24}$

\section{DISCUSSION}

To address why the graphene films doped with $\mathrm{Mes}_{2} \mathrm{~B}^{+}\left[\left(\mathrm{C}_{6} \mathrm{~F}_{5}\right)_{4} \mathrm{~B}\right]^{-}$ are so stable, we performed DFT calculations for the doped graphene- $\left[\left(C_{6} F_{5}\right)_{4} B\right]^{-}$complex, which is formed upon doping (see the Methods section for the computational details). As shown in 
(a)

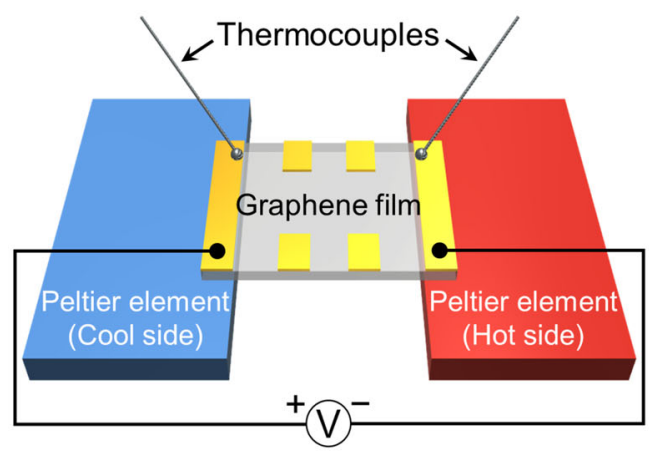

(c)

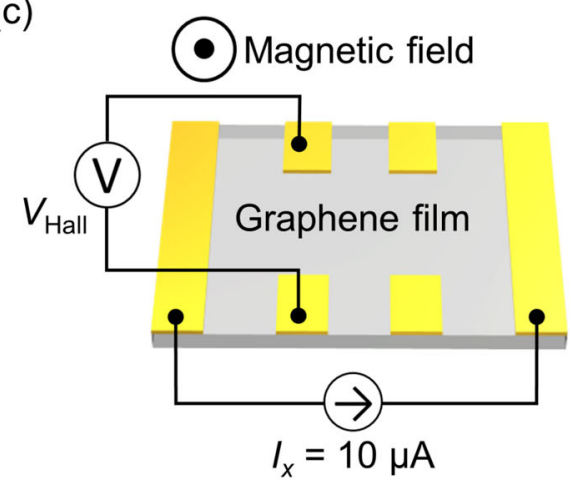

(b)

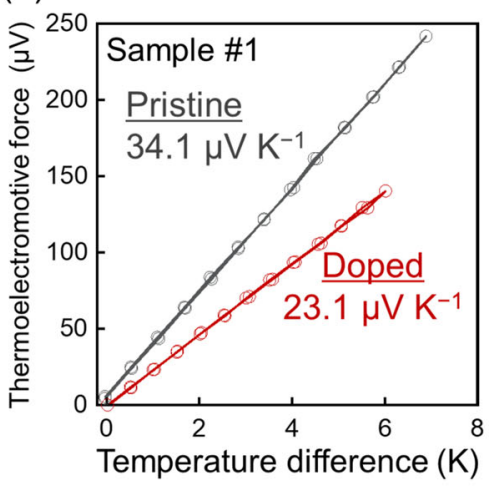

(d) 15

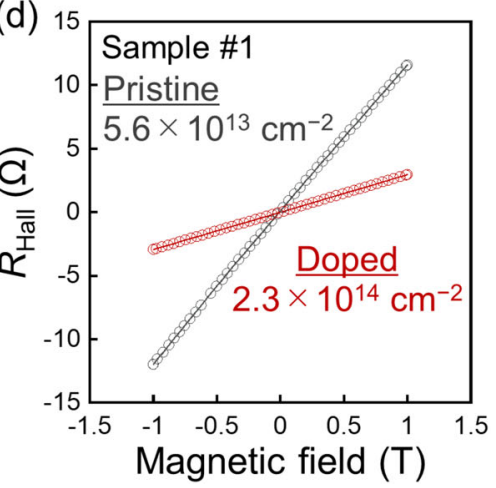

Fig. 4 a Schematic of the experimental setup for the thermopower measurements. $\mathbf{b}$ The thermoelectromotive force and the temperature difference profiles of both pristine and doped graphene samples on a glass substrate (Sample \#1). c Schematic of the experimental setup for the Hall effect measurements. Parameters $I_{x}$ and $V_{\text {Hall }}$ correspond to the constant current and the Hall voltage, respectively. $\mathbf{d}$ The magneticfield dependencies of the Hall resistance $\left(R_{\text {Hall }}=V_{\text {Hall }} / I_{x}\right)$ in both pristine and doped graphene samples on a glass substrate $($ Sample \# 1$)$

Fig. 5a, we used a complex composed of a neutral $\left[\left(\mathrm{C}_{6} \mathrm{~F}_{5}\right)_{4} \mathrm{~B}\right]$ molecule on $7 \times 7$ graphene as the initial structure model for DFT calculations, where the sheet density of $\left[\left(\mathrm{C}_{6} \mathrm{~F}_{5}\right)_{4} \mathrm{~B}\right]$ molecules in this structural model corresponds to $3.9 \times 10^{13} \mathrm{~cm}^{-2}$. After the structure and energy optimization calculation, we carefully checked the resulting charge redistribution in this complex (Fig. $5 \mathrm{~b})$ and confirmed the transfer of 0.6 electron charge from the $7 \times$ 7 graphene to $\left[\left(\mathrm{C}_{6} \mathrm{~F}_{5}\right)_{4} \mathrm{~B}\right]$, resulting in a sheet carrier density of $1.9 \times \times 10^{13} \mathrm{~cm}^{-2}$ in the doped graphene films. Notably, because we did not employ the closest packing structure for $\left[\left(C_{6} F_{5}\right)_{4} B\right]$ molecules in the initial geometry, we expected a higher sheet carrier density for the experimentally doped graphene films.

Figure $5 \mathrm{c}$ presents the results of the energy-band calculations. Importantly, the Fermi energy of the calculated complex is lower than the Dirac point energy of graphene, indicating electron transfer from graphene to the neutral $\left[\left(\mathrm{C}_{6} \mathrm{~F}_{5}\right)_{4} \mathrm{~B}\right]$. To estimate the energy gain due to the formation of the complex of hole-doped graphene and $\left[\left(\mathrm{C}_{6} \mathrm{~F}_{5}\right)_{4} \mathrm{~B}\right]^{-}$, we also calculated the total energy difference between the total energy of the doped $7 \times 7$ graphene$\left[\left(\mathrm{C}_{6} \mathrm{~F}_{5}\right)_{4} \mathrm{~B}\right]^{-}$complex and the sum of the total energies of each constituent, i.e., an isolated $7 \times 7$ graphene and an isolated $\left[\left(C_{6} F_{5}\right)_{4} B\right]$. The calculated binding energy of the molecule to graphene is $573 \mathrm{meV} /$ molecule, which is significantly larger than the energy of thermal excitation at room temperature $(\sim 26 \mathrm{meV})$. Consequently, the back electron transfer from $\left[\left(\mathrm{C}_{6} \mathrm{~F}_{5}\right)_{4} \mathrm{~B}\right]^{-}$to holedoped graphene is very unlikely, and hole-doped graphene films with $\mathrm{Mes}_{2} \mathrm{~B}^{+}\left[\left(\mathrm{C}_{6} \mathrm{~F}_{5}\right)_{4} \mathrm{~B}\right]^{-}$should be stable at room temperature. Moreover, the large binding energy is ascribed to the Coulomb interaction between the hole carriers in graphene and the electron carriers in the molecule caused by the charge transfer between them, which substantially stabilizes $\left[\left(\mathrm{C}_{6} \mathrm{~F}_{5}\right)_{4} \mathrm{~B}\right]^{-}$on the graphene surface, and is therefore advantageous for introducing an additional rinsing step after the doping process. Therefore, our theoretical calculation predicts that $\mathrm{Mes}_{2} \mathrm{~B}^{+}\left[\left(\mathrm{C}_{6} \mathrm{~F}_{5}\right)_{4} \mathrm{~B}\right]^{-}$is a superb molecular hole dopant for graphene thin films, which is in reasonable agreement with experimental results such as the sheet resistivity, transmittance, XPS, thermopower, and the Hall effect measurements.

In summary, the efficient hole doping of graphene using the salt of two-coordinate boron cation $\mathrm{Mes}_{2} \mathrm{~B}^{+}$with counter anion $\left[\left(C_{6} F_{5}\right)_{4} B\right]^{-}$has been demonstrated in this study. The high hole density of $\sim 2.5 \times 10^{14} \mathrm{~cm}^{-2}$ was achieved within the 4-s solutionbased doping method. The improved conduction was maintained for at least 31 days in air without a substantial change. Moreover, the transmittance reduction by doping was only $0.4 \%$. Notably, the highly transparent graphene films with low sheet resistance were reproducibly obtained by a combination of hole doping with $\mathrm{Mes}_{2} \mathrm{~B}^{+}\left[\left(\mathrm{C}_{6} \mathrm{~F}_{5}\right)_{4} \mathrm{~B}\right]^{-}$and additional solvent rinsing with ODCB. Finally, we successfully fabricated OPVs using the Mes $_{2} \mathrm{~B}^{+}\left[\left(\mathrm{C}_{6} \mathrm{~F}_{5}\right)_{4} \mathrm{~B}\right]^{-}$-doped graphene as electrodes, where the doped graphene electrodes positively affected the OPV performance.

\section{METHODS}

Synthesis of CVD-grown graphene films and solution-based doping with $\mathrm{Mes}_{2} \mathrm{~B}^{+}\left[\left(\mathrm{C}_{6} \mathrm{~F}_{5}\right)_{4} \mathrm{~B}\right]$

Polycrystalline $\mathrm{Cu}$ foils $(23 \mathrm{~cm} \times 20 \mathrm{~cm} \times 33-\mu \mathrm{m}$ thick) were treated with an $\mathrm{Ar} / \mathrm{H}_{2}$ plasma at $5 \mathrm{~Pa}$ for $20 \mathrm{~min}$ to clean their surfaces before the CVD process. After cleaning, we performed CVD (CVD conditions: $3 \mathrm{~Pa}, \mathrm{CH}_{4} / \mathrm{Ar} /$ $\mathrm{H}_{2}=30 / 20 / 10$ standard cubic centimeters per minute, and $4.5 \mathrm{~kW}$ for each microwave generator). The synthesized graphene films were transferred onto two types of substrate, PET (188 $\mu \mathrm{m}$ thick) and glass ( $1 \mathrm{~mm}$ thick). 
(a)

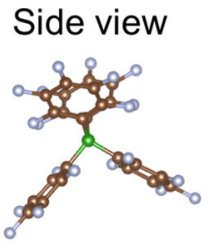

$\infty=\infty-\infty=\infty=\infty=\infty-\infty$

(b) Side view

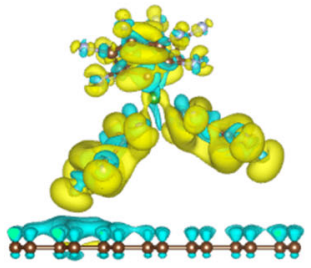

Top view

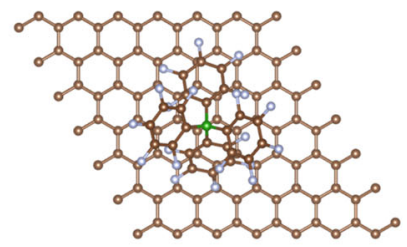

Top view

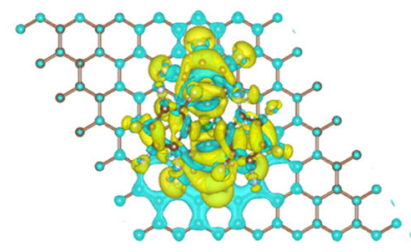

(c)

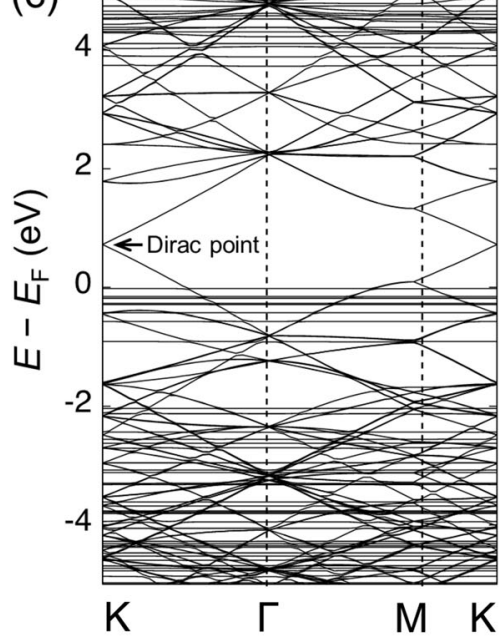

Fig. 5 a The initial geometry of a neutral $\left[\left(\mathrm{C}_{6} \mathrm{~F}_{5}\right)_{4} \mathrm{~B}\right]$ molecule adsorbed onto $7 \times 7$ graphene as the structure model for DFT calculations. $\mathbf{b}$ Isosurfaces of the charge-redistributed $\left[\left(\mathrm{C}_{6} \mathrm{~F}_{5}\right)_{4} \mathrm{~B}\right]^{-}-7 \times 7$ graphene complex. Yellow and blue isosurfaces correspond to the electron-rich and hole-rich areas, respectively. c Calculated energy-band structure of the $\left[\left(C_{6} F_{5}\right)_{4} B\right]^{-}-7 \times 7$ graphene complex. The vertical axis shows the relative energy from the Fermi level

The following process was performed inside a $\mathrm{N}_{2}$-filled glove box with $<1 \mathrm{ppm}$ oxygen and moisture at room temperature. A saturated ODCB solution of $\mathrm{Mes}_{2} \mathrm{~B}^{+}\left[\left(\mathrm{C}_{6} \mathrm{~F}_{5}\right)_{4} \mathrm{~B}\right]^{-}(30 \mathrm{mg} / \mathrm{mL})$ was prepared for doping. ${ }^{31,32}$ The graphene samples were immersed in the ODCB solution of $\operatorname{Mes}_{2} B^{+}\left[\left(C_{6} F_{5}\right)_{4} B\right]^{-}$for 1 min. The graphene samples were then taken from the ODCB solution and rinsed with dry ODCB for $\sim 10 \mathrm{~s}$ to clean the surface of the samples. Finally, the doped graphene samples were annealed at $60^{\circ} \mathrm{C}$ for $10 \mathrm{~min}$ on a hotplate to remove any residual ODCB.

\section{Sample characterization}

Raman spectra were obtained using a Raman spectrophotometer (JASCO, NRS-5000) with a 532-nm laser excitation. For the test of the carrier-doping capability of $\mathrm{Mes}_{2} \mathrm{~B}^{+}\left[\left(\mathrm{C}_{6} \mathrm{~F}_{5}\right)_{4} \mathrm{~B}\right]^{-}$inside a $\mathrm{N}_{2}$-filled glove box, the four-probe sheet resistances of graphene samples were measured at a constant voltage of $500 \mathrm{mV}$ using a semiconductor parameter analyzer (Agilent, B2902A). In ambient air, the four-probe sheet resistances of graphene samples were collected without any atmospheric regulation. Graphene samples were wired in series with a source meter (Agilent, B2902A). We applied a current ranging from -10 to $10 \mu \mathrm{A}$, and the voltage drops in each sample were monitored with a nanovoltmeter (Keysight, $34420 \mathrm{~A}$ ). The optical transmission spectra of graphene samples were collected using a UV-Vis-NIR spectrophotometer (Agilent, Cary 5000). XPS was performed under vacuum using $\mathrm{Mg} \mathrm{Ka}$ radiation (JEOL, JPS-9010TR). The thermopower was measured in a $\mathrm{N}_{2}$-filled glove box using a previously reported measurement setup. ${ }^{43,44}$ We placed a graphene sample on two Peltier elements (Laird Technologies, CP1.0-31-05L) wired in series with a DC power supply (Keysight, E3642A) to induce a temperature difference. In addition, two alumel-chromel thermocouples (SAKAGUCHI EH VOC CORP., TCKT0051) were placed on the surface of the sample. The temperature difference and thermoelectromotive force were obtained using a voltmeter (Keysight, 34420A) and temperature controller (Lakeshore, model 335), respectively. The Hall effect measurements were performed in a physical properties measurement system (Quantum Design, PPMS). The temperature was controlled at $300 \mathrm{~K}$, and constant currents $\left(I_{x}\right)$ of 1 and $10 \mu \mathrm{A}$ were applied for pristine and doped graphene samples, respectively. $R_{\text {Hall }}$ was calculated as the equation of $R_{\text {Hall }}=V_{\text {Hall }} / I_{x}$. The magnetic field was slowly swept perpendicular to both the sample and the current direction between -1 and $1 \mathrm{~T}$.

\section{Computational details of energy-band calculations}

Theoretical calculations were performed using the DFT method ${ }^{45}$ with the generalized gradient approximation (GGA-PBE) ${ }^{46}$ and van der Waals (vdW) corrections $(\mathrm{vdW}-\mathrm{DF} 2-\mathrm{C} 09)^{47}$ implemented in the STATE package. ${ }^{48}$ Vanderbilt ultrasoft pseudopotentials were also used to describe electron-ion interactions. ${ }^{49}$ Valence and deficit charge densities were expanded in terms of the plane-wave basis set with cutoff energies of 25 and 225 Ry, respectively. As an initial geometry, a neutral $\left[\left(C_{6} F_{5}\right)_{4} B\right]$ molecule was placed on $7 \times 7$ lateral supercell of single-layer graphene. To exclude the unphysical dipole interaction due to the charge transfer between graphene and $\left(\mathrm{C}_{6} \mathrm{~F}_{5}\right)_{4} \mathrm{~B}$, we imposed an open boundary condition normal to the graphene layer with a vacuum spacing of $0.5 \mathrm{~nm}$ using the effective screening medium method. ${ }^{50}$ For the Brillouin zone integration, $\Gamma$ point sampling was used.

\section{DATA AVAILABILITY}

All data related to the manuscript is available on request from the corresponding author.

\section{ACKNOWLEDGEMENTS}

Ka.K. acknowledges the Leading Graduate Program in Science and Engineering, Waseda University from the Ministry of Education, Culture, Sports, Science and Technology in Japan (MEXT). T.T. is partially supported by JSPS KAKENHI (Grant Numbers JP26102012, JP25000003, and JP17H01069) and JST CREST (Grant Number JPMJCR1715). H.O. is supported by JSPS KAKENHI (JP17H01314) and the Asahi Glass Foundation. T.F. is supported by Grant-in-Aid for Scientific Research on Innovative Areas from MEXT (JP26102008 " $\pi$-System Figuration"). Y.S. is supported by Asahi Grass Foundation. This work was performed under the Cooperative Research Program of "Network Joint Research Center for Materials and Devices" from MEXT.

\section{AUTHOR CONTRIBUTIONS}

T.T. conceived the project and designed the experiments. Ka.K. performed the fourprobe sheet resistance, transmittance, X-ray photoelectron spectroscopy, thermopower, and the Hall effect measurements and carried out the data analysis. N.T., Y.S., and T.F. contributed to the synthesizing of $\operatorname{Mes}_{2} B^{+}\left[\left(C_{6} F_{5}\right)_{4} B\right]^{-}$. M.I. and M.H. performed the surface wave plasma CVD of large-area graphene films. H.O. assisted in the preparation of the thermopower measurement system. M.M. and S.O. performed DFT calculations. Ke.K. and H.A. prepared single-layer graphene and double-layer graphene films by a thermal CVD method. I.J. and Y.M. fabricated organic photovoltaic cells with graphene electrodes and analyzed the data. All the authors interpreted the data, commented on the manuscript, and agreed with the results and conclusions.

\section{ADDITIONAL INFORMATION}

Supplementary information accompanies the paper on the npj 2D Materials and Applications website (https://doi.org/10.1038/s41699-019-0090-x).

Competing interests: The authors declare no competing interests. 
Publisher's note: Springer Nature remains neutral with regard to jurisdictional claims in published maps and institutional affiliations.

\section{REFERENCES}

1. Kim, K. S. et al. Large-scale pattern growth of graphene films for stretchable transparent electrodes. Nature 457, 706-710 (2009).

2. Lee, Y. et al. Wafer-scale synthesis and transfer of graphene films. Nano. Lett. 10, 490-493 (2010).

3. Bae, S. et al. Roll-to-roll production of 30-inch graphene films for transparent electrodes. Nat. Nanotechnol. 5, 574-578 (2010).

4. Kim, J. et al. Low-temperature synthesis of large-area graphene-based transparent conductive films using surface wave plasma chemical vapor deposition. Appl. Phys. Lett. 98, 091502 (2011).

5. Reddy, A. L. M. et al. Synthesis of nitrogen-doped graphene films for lithium battery application. ACS Nano 4, 6337-6342 (2010).

6. Rana, K. et al. Highly conductive freestanding graphene films as anode current collectors for flexible lithium-ion batteries. ACS Appl. Mater. Interfaces 6, 11158-11166 (2014).

7. Cao, X. et al. Preparation of novel 3D graphene networks for supercapacitor applications. Small 7, 3163-3168 (2011)

8. Dong, X. C. et al. 3D Graphene-cobalt oxide electrode for high-performance supercapacitor and enzymeless glucose detection. ACS Nano 6, 3206-3213 (2012).

9. Kim, K. et al. Ultrathin organic solar cells with graphene doped by ferroelectric polarization. ACS Appl. Mater. Interfaces 6, 3299-3304 (2014).

10. Liu, Z. et al. Package-free flexible organic solar cells with graphene top electrodes. Adv. Mater. 25, 4296-4301 (2013).

11. De Arco, L. G. et al. Continuous, highly flexible, and transparent graphene films by chemical vapor deposition for organic photovoltaics. ACS Nano 4, 2865-2873 (2010).

12. Wang, C. et al. Electrochemical properties of graphene paper electrodes used in lithium batteries. Chem. Mater. 21, 2604-2606 (2009).

13. Lee, C. et al. Measurement of the elastic properties and intrinsic strength of monolayer graphene. Science 321, 385-388 (2008).

14. Rasool, H. I. et al. Measurement of the intrinsic strength of crystalline and polycrystalline graphene. Nat. Commun. 4, 2811 (2013).

15. Geim, A. K. \& Novoselov, K. S. The rise of graphene. Nat. Mater. 6, 183-191 (2007)

16. Morozov, S. V. et al. Giant intrinsic carrier mobilities in graphene and its bilayer. Phys. Rev. Lett. 100, 016602 (2008).

17. Zhang, Y. et al. Review of chemical vapor deposition of graphene and related applications. Acc. Chem. Res. 46, 2329-2339 (2013).

18. Kim, K. K. et al. Enhancing the conductivity of transparent graphene films via doping. Nanotechnology 21, 285205 (2010).

19. Tongay, S. et al. Stable hole doping of graphene for low electrical resistance and high optical transparency. Nanotechnology 22, 425701 (2011).

20. Kwon, K. C. et al. Increase work function in few-layer graphene sheets via metal chloride doping. Adv. Funct. Mater. 22, 4724-4731 (2012).

21. Shin, D. H. et al. Annealing effect on the characteristics of $\mathrm{AuCl}_{3}$-doped graphene. J. Appl. Phys. 113, 064305 (2013).

22. Shin, D. H. et al. Enhancement of the effectiveness of graphene as a transparent conductive electrode by $\mathrm{AgNO}_{3}$ doping. Nanotechnology 25, 125701 (2014).

23. Jang, C. W. et al. Degradation reduction and stability enhancement of p-type graphene by $\mathrm{RhCl}_{3}$ doping. J. Alloy. Comp. 621, 1-6 (2015).

24. Mansour, A. E. et al. Facile doping and work-function modification of few-layer graphene using molecular oxidants and reductants. Adv. Funct. Mater. 27, 1602004 (2017).

25. Chandramohan, S. et al. A comparison of various surface charge transfer hole doping of graphene grown by chemical vapour deposition. Appl. Surf. Sci. 418 258-263 (2017).

26. Khrapach, I. et al. Novel highly conductive and transparent graphene-based conductors. Adv. Mater. 24, 2844-2849 (2012).

27. Mansour, A. E. et al. Hybrid doping of few-layer graphene via a combination of intercalation and surface doping. ACS Appl. Mater. Interfaces 9, 20020-20028 (2017).
28. Kinoshita, $\mathrm{H}$. et al. Highly conductive and transparent large-area bilayer graphene realized by $\mathrm{MoCl}_{5}$ intercalation. Adv. Mater. 29, 1702141 (2017).

29. Jeon, I. et al. Carbon nanotubes versus graphene as flexible transparent electrodes in inverted perovskite solar cells. J. Phys. Chem. Lett. 8, 5395-5401 (2017).

30. Shoji, Y. et al. A two-coordinate boron cation featuring $\mathrm{C}^{-} \mathrm{B}^{+}-\mathrm{C}$ bonding. Nat. Chem. 6, 498-508 (2014).

31. Funahashi, K. et al. Highly air- and moisture-stable hole-doped carbon nanotube films achieved using boron-based oxidant. Appl. Phys. Express 10, 035101 (2017).

32. Matsuoka, $\mathrm{H}$. et al. Chemical hole doping into large-area transition metal dichalcogenide monolayers using boron-based oxidant. Jpn. J. Appl. Phys. 57, 02CB15 (2018).

33. Shoji, Y. et al. The molecular and electronic structures of a thioaroyl cation formed by borinium ion-mediated $\mathrm{C}=\mathrm{S}$ double bond cleavage of $\mathrm{CS}_{2}$. Chem. Commun. 51, 13342-13345 (2015).

34. Knapp, C. In Comprehensive Inorganic Chemistry II Vol. 1 (eds Reedijk, J. \& Poeppelmeier, K.) 651 (Elsevier, Amsterdam, 2013).

35. Kim, J. et al. Large-area surface wave plasmas using microwave multi-slot antennas for nanocrystalline diamond film deposition. Plasma Sources Sci. Technol. 19, 015003 (2010).

36. Jeon, l. et al. Room temperature-processed inverted organic solar cells using high working-pressure-sputtered ZnO films. J. Mater. Chem. A 4, 18763 (2016).

37. Javier, D. et al. Separation of the $\mathrm{sp} 3$ and sp2 components in the C1s photoemission spectra of amorphous carbon films. Phys. Rev. B 54, 8064 (1996).

38. Gelius, U. et al. Molecular spectroscopy by means of ESCA III. Carbon Compd. Phys. Scr. 2, 70-80 (1970)

39. Clark, D. T. et al. An ESCA study of the molecular core binding energies of the fluorobenzenes. J. Electron Spectrosc. 1, 227-250 (1972).

40. Paniagua, S. A. et al. Production of heavily $n$ - and $p$-doped CVD graphene with solution-processed redox-active metal-organic species. Mater. Horiz. 1, 111-115 (2014).

41. Cançado, L. G. et al. Quantifying defects in graphene via raman spectroscopy at different excitation energies. Nano. Lett. 11, 3190-3196 (2011).

42. Kittel, C. Introduction to Solid State Physics 7th edn (Wiley, New York, 1996).

43. $\mathrm{Pu}, \mathrm{J}$. et al. Enhanced thermoelectric power in two-dimensional transition metal dichalcogenide monolayers. Phys. Rev. B 94, 014312 (2016).

44. Ohta, H. et al. Unusually large enhancement of thermopower in an electric field induced two-dimensional electron gas. Adv. Mater. 24, 740-744 (2012).

45. Kohn, W. \& Sham, L. J. Self-consistent equations including exchange and correlation effects. Phys. Rev. 140, A1133-A1138 (1965).

46. Perdew, J. P. et al. Generalized gradient approximation made simple. Phys. Rev. Lett. 77, 3865-3868 (1996).

47. Hamada, I. \& Otani, M. Comparative van der Waals density-functional study of graphene on metal surfaces. Phys. Rev. B 82, 153412 (2010).

48. Morikawa, Y. et al. Theoretical study of hydrogenation process of formate on clean and An deposited Cu(111) surfaces. Appl. Surf. Sci. 169-170, 11-15 (2001)

49. Vanderbilt, D. Soft self-consistent pseudopotentials in a generalized eigenvalue formalism. Phys. Rev. B 41, 7892-7895 (1990).

50. Otani, M. \& Sugino, O. First-principles calculations of charged surfaces and interfaces: A plane-wave nonrepeated slab approach. Phys. Rev. B 73, 115407 (2006).

(i) Open Access This article is licensed under a Creative Commons Attribution 4.0 International License, which permits use, sharing, adaptation, distribution and reproduction in any medium or format, as long as you give appropriate credit to the original author(s) and the source, provide a link to the Creative Commons license, and indicate if changes were made. The images or other third party material in this article are included in the article's Creative Commons license, unless indicated otherwise in a credit line to the material. If material is not included in the article's Creative Commons license and your intended use is not permitted by statutory regulation or exceeds the permitted use, you will need to obtain permission directly from the copyright holder. To view a copy of this license, visit http://creativecommons. org/licenses/by/4.0/.

(c) The Author(s) 2019 